\title{
Quantum Benchmark via an Uncertainty Product of Canonical Variables
}

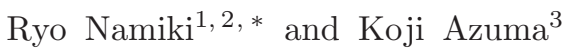 \\ ${ }^{1}$ Department of Physics, Graduate School of Science, Kyoto University, Kyoto 606-8502, Japan \\ ${ }^{2}$ Institute for Quantum Computing and Department of Physics and Astronomy, \\ University of Waterloo, Waterloo, Ontario, N2L 3G1, Canada \\ ${ }^{3}$ NTT Basic Research Laboratories, NTT Corporation, \\ 3-1 Morinosato Wakamiya, Atsugi, Kanagawa 243-0198, Japan
}

(Dated: June 26, 2018)

\begin{abstract}
We present an uncertainty-relation-type quantum benchmark for continuous-variable (CV) quantum channels that works with an input ensemble of Gaussian distributed coherent states and homodyne measurements. It determines an optimal trade-off relation between canonical quadrature noises unbeatable by entanglement breaking channels and refines the notion of two quantum duties introduced in the original papers of CV quantum teleportation. It can verify the quantum-domain performance for all one-mode Gaussian channels. We also address the case of stochastic channels and the effect of asymmetric gains.
\end{abstract}

The quantum benchmarks (QB) [1-8] provide a fundamental criterion for experimental success of quantum gates and channels by eliminating the possibility that the physical process is described by an entanglement breaking (EB) channel [9]. This criterion ensures that the channel is capable of transmitting quantum entanglement and draws a firm bottom line on implementation of quantum gates based on the notion of entanglement. The QBs enable us to demonstrate an advantage of entanglement in quantum teleportation process and a non-classical performance in quantum memories [4, 10 12]. They also give a prerequisite for quantum key distribution [13 16]. A common framework is to observe a response of the gate operation for a set of non-orthogonal input states. Currently, the majority of QBs have been given in terms of an average fidelity [12, 17, 18] and a main theoretical task is to determine the classical limit of the fidelity achieved by EB channels. By surpassing such a classical limit, one can verify that the channel is in the quantum domain, namely, not an EB channel.

Although the fidelity is a central tool to certify the performance of quantum gates in quantum information science, there has been a general interest to invoke the canonical uncertainty relation or quadrature noises in evaluating continuous-variable (CV) quantum channels 10, 11, 13, 19 23]. In such approaches, an incoherence of the gate operation can be intuitively explained by the amount of excess quadrature noises above the shot noise limit assuming the transmission of coherent states or minimum uncertainty states. Measurements of canonical quadratures are also favorable in many of experiments in quantum information with light and atoms [19, 20, 24 26]. In addition, it recalls a primary question in quantum physics whether a simple trade-off relation holds between the pair of noises provided that two of non-commuting observables are measured [27, 28]. However, such an insightful aspect has little been addressed in quantum benchmarking.

One can find an outstanding puzzle on the property of quadrature noises induced by EB maps. The original papers of CV quantum teleportation [10] suggested that, to validate an entanglement assistance, the amount of excess noises has to be smaller than two units of the shot noise, referred to as two quantum duties (two quduties). There is a famous theorem that a single shot-noise unit of excess noise is unavoidable in the simultaneous measurement of canonical quadratures [27 29]. By associating this theorem with another theorem [9] that any EB channel can be described as a measurement and a following state preparation, it is fascinating to interpret each of the measurement and the state preparation processes as being responsible for a single shot-noise penalty [30]. However, this interpretation is inaccurate because the penalty of two quduties has not been proven to be a classical limit unbeatable by any EB channel until now. To this end, the notion of two quduties is missing rigorous links to QBs although it sounds highly interesting 31].

On the contrary, a practical CV QB has been established by determining the classical limit fidelity for an input ensemble using coherent states with a Gaussian prior [4, 5, 21]. This input ensemble called the Gaussian distributed coherent states gives a modest experimental setting to observe an effectively linear gate response in the CV space where the Gaussian prior suppresses the contribution of unfeasibly high-energy input states. Although it is customary to describe a normal CV channel with a gain and excess noise terms of canonical quadrature operators 24, 25], the scope of the CV QB had initially been limited for unit-gain channels corresponding to unitary action of $\mathrm{CV}$ channels [4]. It was then extended for non-unit-gain channels in order to deal with an important class of non-unitary processes such as lossy channels and amplification channels [5]. This extension is sufficient to detect all one-mode Gaussian channels in the quantum domain [5] similarly to the case of the famous sum criterion for CV entanglement that witnesses all two-mode Gaussian entanglement [32, 33]. Recently, another extension has been made to serve for probabilistic operations 34]. Hence, the fidelity-based QB provides a standard method for estimating CV quantum operations as well as channels. On the other hand, proving a CV process with the ensemble of Gaussian distributed coherent states could be a more general idea applicable to varieties of measurement scenarios other than the fidelity. However, it is an open question whether such a 
setting finds a significant utility besides the fidelity-based method.

In this Letter, we present an EB limit in a product form of canonical noises averaged over Gaussian distributed coherent states. It offers a QB that generally explains an optimal trade-off relation between the canonical variables rendered by EB maps and refines the notion of two quduties. Our QB is shown to be sufficient for detecting all one-mode Gaussian channels in the quantum domain. We also generalize our QB to deal with probabilistic operations and an asymmetry of quadrature gains. Our results almost repeat the fidelity-based achievements but demonstrate a fundamental role of canonical variables to observe genuine quantum coherence in a physical process.

Our goal is to derive a bound from the first and second moments of canonical variables for output states of a given channel $\mathcal{E}$ by assuming input of coherent states. We start with the product separable condition 35] in a normalized form [36]: Any separable state $J_{A B}$ satisfies

$$
\begin{aligned}
& \operatorname{Tr}\left[\left(u \hat{x}_{A}-v \hat{x}_{B}\right)^{2} J\right] \operatorname{Tr}\left[\left(u \hat{p}_{A}+v \hat{p}_{B}\right)^{2} J\right] \\
\geq & \left\langle\Delta^{2}\left(u \hat{x}_{A}-v \hat{x}_{B}\right)\right\rangle_{J}\left\langle\Delta^{2}\left(u \hat{p}_{A}+v \hat{p}_{B}\right)\right\rangle_{J} \geq \frac{1}{4},
\end{aligned}
$$

where $(u, v)$ is a real vector with $u^{2}+v^{2}=1$ and the canonical variables satisfy $\left[\hat{x}_{A}, \hat{p}_{A}\right]=\left[\hat{x}_{B}, \hat{p}_{B}\right]=i$. The first inequality is due to the property of variances, $\left\langle\hat{o}^{2}\right\rangle \geq$ $\left\langle\Delta^{2} \hat{o}\right\rangle$.

Let us write $\hat{x}_{B}=\left(\hat{b}+\hat{b}^{\dagger}\right) / \sqrt{2}$ and $\hat{p}_{B}=\left(\hat{b}-\hat{b}^{\dagger}\right) /(\sqrt{2} i)$. From the cyclic property of the trace we have

$$
\begin{aligned}
\operatorname{Tr}_{B}\left[\hat{x}_{B} J\right] & =\operatorname{Tr}_{B}\left[\hat{b}^{\dagger} J+J \hat{b}\right] / \sqrt{2}, \\
\operatorname{Tr}_{B}\left[\hat{x}_{B}^{2} J\right] & \left.=\operatorname{Tr}_{B}\left[\hat{b}^{\dagger 2} J+J \hat{b}^{2}+2 \hat{b}^{\dagger} J \hat{b}-J\right)\right] / 2, \\
\operatorname{Tr}_{B}\left[\hat{p}_{B} J\right] & =i \operatorname{Tr}_{B}\left[\hat{b}^{\dagger} J-J \hat{b}\right] / \sqrt{2}, \\
\operatorname{Tr}_{B}\left[\hat{p}_{B}^{2} J\right] & =-\operatorname{Tr}_{B}\left[\hat{b}^{\dagger 2} J+J \hat{b}^{2}-2 \hat{b}^{\dagger} J \hat{b}+J\right] / 2 .
\end{aligned}
$$

Here, $\operatorname{Tr}_{A(B)}$ denotes the partial trace over subsystem $A(B)$. Let us write the partial trace as $\operatorname{Tr}_{B}[\cdot] \rightarrow$ $\int\left\langle\alpha^{*}|\cdot| \alpha^{*}\right\rangle_{B} d^{2} \alpha / \pi$ by the completeness relation for coherent states. Then, the property of the coherent state, $\hat{b}\left|\alpha^{*}\right\rangle_{B}=\alpha^{*}\left|\alpha^{*}\right\rangle_{B}$, enables us to show

$$
\begin{aligned}
& \operatorname{Tr}_{B}\left[\hat{x}_{B} J\right]=\int x_{\alpha}\left\langle\alpha^{*}|J| \alpha^{*}\right\rangle_{B} \frac{d^{2} \alpha}{\pi}, \\
& \operatorname{Tr}_{B}\left[\hat{x}_{B}^{2} J\right]=\int x_{\alpha}^{2}\left\langle\alpha^{*}|J| \alpha^{*}\right\rangle_{B} \frac{d^{2} \alpha}{\pi}-\frac{J_{A}}{2}, \\
& \operatorname{Tr}_{B}\left[\hat{p}_{B} J\right]=-\int p_{\alpha}\left\langle\alpha^{*}|J| \alpha^{*}\right\rangle_{B} \frac{d^{2} \alpha}{\pi}, \\
& \operatorname{Tr}_{B}\left[\hat{p}_{B}^{2} J\right]=\int p_{\alpha}^{2}\left\langle\alpha^{*}|J| \alpha^{*}\right\rangle_{B} \frac{d^{2} \alpha}{\pi}-\frac{J_{A}}{2},
\end{aligned}
$$

where $J_{A}=\operatorname{Tr}_{B}[J]$ and we use a shorthand notation of the mean quadratures of a coherent state as

$$
x_{\alpha}:=\langle\alpha|\hat{x}| \alpha\rangle=\frac{\alpha+\alpha^{*}}{\sqrt{2}}, p_{\alpha}:=\langle\alpha|\hat{p}| \alpha\rangle=\frac{\alpha-\alpha^{*}}{\sqrt{2} i} .
$$

By substituting Eqs. (3) into the first line of Eq. (11) we obtain the following Lemma.
Lemma.-Any separable state $J_{A B}$ has to satisfy

$\prod_{z \in\{x, p\}}\left[\operatorname{Tr}_{A} \int\left(u \hat{z}_{A}-v z_{\alpha}\right)^{2}\left\langle\alpha^{*}|J| \alpha^{*}\right\rangle_{B} \frac{d^{2} \alpha}{\pi}-\frac{v^{2}}{2}\right] \geq \frac{1}{4}$.

We may call this Lemma the hybrid separable condition because it can be seen as an entanglement detection scheme where homodyne and heterodyne measurements are respectively performed on subsystems $A$ and $B$ [37]. Our main result is the following Proposition.

Proposition.-For any $\lambda>0$ and $\eta>0$, any EB channel $\mathcal{E}$ satisfies the uncertainty limit (See Fig. 1)

$$
\left[\bar{V}_{x}-\frac{\eta}{2(1+\lambda)}\right]\left[\bar{V}_{p}-\frac{\eta}{2(1+\lambda)}\right] \geq \frac{1}{4}\left(1+\frac{\eta}{1+\lambda}\right)^{2}
$$

where the mean square deviation is defined through

$$
\bar{V}_{z}=\bar{V}_{z}(\eta, \lambda):=\operatorname{Tr} \int p_{\lambda}(\alpha)\left(\hat{z}-\sqrt{\eta} z_{\alpha}\right)^{2} \mathcal{E}\left(\rho_{\alpha}\right) d^{2} \alpha,
$$

with $\rho_{\alpha}:=|\alpha\rangle\langle\alpha|$ and the prior Gaussian distribution

$$
p_{\lambda}(\alpha):=\frac{\lambda}{\pi} \exp \left(-\lambda|\alpha|^{2}\right) .
$$

This prior enables us to neglect the contribution of high energy states with $|\alpha|^{2} \gg \lambda^{-1}$ and represents a flat distribution in the limit $\lambda \rightarrow 0$. As we will see, the gain factor $\eta$ and the input ensemble of Gaussian distributed coherent states $\left\{p_{\lambda}(\alpha), \rho_{\alpha}\right\}_{\alpha \in \mathbb{C}}$ are naturally introduced from a simple entanglement detection scenario which uses our Lemma on a two-mode state given by applying a quantum channel $\mathcal{E}$ to a two-mode squeezed state. Moreover, the pair $\left(\bar{V}_{x}, \bar{V}_{p}\right)$ essentially comes from the quadrature correlations in Eq. (1) and represents the noise terms of $\mathcal{E}$. It can be directly measured by homodyne detection on the output state $\mathcal{E}\left(\rho_{\alpha}\right)$. Note that Eq. (6) corresponds to the canonical uncertainty relation when $\eta=0$.

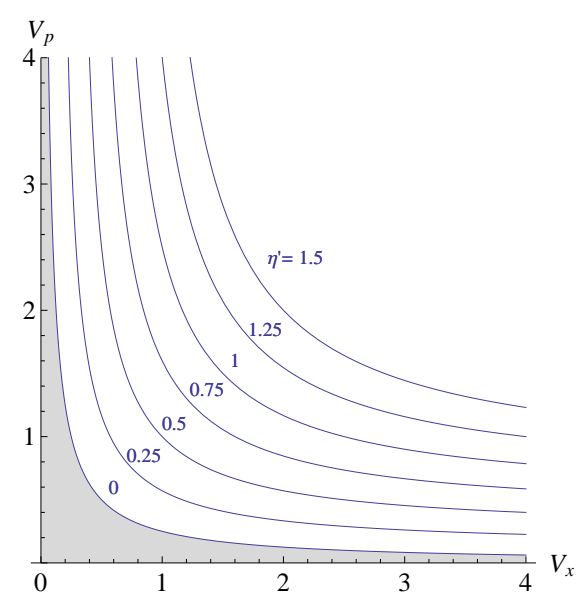

FIG. 1: Entanglement breaking limit for quadrature noises in Eq. (6) for a set of a normalized gain $\eta^{\prime}=\eta /(1+\lambda)$. The case of $\eta=0$ retrieves the minimum uncertainty curve, and the shade represents physically unaccessible regime.

Proof of Proposition. - Suppose that the bipartite state $J$ is prepared by the action of a channel $\mathcal{E}$ as [38, 39]

$$
J=\mathcal{E}_{A} \otimes I_{B}\left(\left|\psi_{\xi}\right\rangle\left\langle\psi_{\xi}\right|\right)
$$


where $\left|\psi_{\xi}\right\rangle=\sqrt{1-\xi^{2}} \sum_{n=0}^{\infty} \xi^{n}|n\rangle|n\rangle$ with $\xi \in(0,1)$ is a two-mode squeezed state and $I$ represents the identity process. This implies

$$
\left\langle\alpha^{*}|J| \alpha^{*}\right\rangle_{B}=\left(1-\xi^{2}\right) e^{-\left(1-\xi^{2}\right)|\alpha|^{2}} \mathcal{E}_{A}\left(\rho_{\xi \alpha}\right) .
$$

From Eqs. (3), (7), (8), and (10) we can write

$$
\begin{aligned}
& \operatorname{Tr}_{A} \int\left(u \hat{z}_{A}-v z_{\alpha}\right)^{2}\left\langle\alpha^{*}|J| \alpha^{*}\right\rangle_{B} \frac{d^{2} \alpha}{\pi} \\
= & u^{2}\left[\operatorname{Tr}_{A} \int p_{\lambda}(\alpha)\left(\hat{z}_{A}-\sqrt{\eta} z_{\alpha}\right)^{2} \mathcal{E}_{A}\left(\rho_{\alpha}\right) d^{2} \alpha\right]=u^{2} \bar{V}_{z},
\end{aligned}
$$

where $z \in\{x, p\}$, the variable of the integration is rescaled as $\xi \alpha \rightarrow \alpha$, and new parameters are given by

$$
\lambda=\frac{1-\xi^{2}}{\xi^{2}}>0, \eta=\frac{1}{\xi^{2}}\left(\frac{v}{u}\right)^{2} \geq 0 .
$$

If we eliminate $\xi$ in Eqs. (12), we have

$$
\frac{1}{u^{2}}=\left(1+\frac{\eta}{1+\lambda}\right), \text { or equivalently } \frac{v^{2}}{u^{2}}=\frac{\eta}{1+\lambda} .
$$

Now, suppose that $\mathcal{E}$ is an EB channel. Then $J$ of Eq. (9) is a separable state, and we can use Lemma. By substituting Eq. (11) into Eq. (5) we have

$$
u^{4}\left(\bar{V}_{x}-\frac{(v / u)^{2}}{2}\right)\left(\bar{V}_{p}-\frac{(v / u)^{2}}{2}\right) \geq \frac{1}{4} .
$$

Finally, substituting Eq. (13) into this expression we obtain Eq. (6).

Any violation of the condition of Eq. (6) implies that the channel cannot be simulated by EB channels, and it establishes a QB to verify the quantum-domain process with the input ensemble of Gaussian distributed coherent states and normal quadrature measurements. Main implication of our QB is the followings: For unit gain $\eta=1$ and completely unknown coherent states $\lambda \rightarrow 0$, Eq. (6) reduces to $\left(\bar{V}_{x}-V_{0}\right)\left(\bar{V}_{p}-V_{0}\right) \geq\left(2 V_{0}\right)^{2}$ where $V_{0}:=\left\langle\Delta^{2} \hat{z}\right\rangle_{\rho_{\alpha}}=1 / 2$ is the variance of coherent states or the shot noise. This implies $\bar{V}_{z}-V_{0} \geq 0$ represents the extra noise added by the channel $\mathcal{E}$. Then, the inequality $\left(\bar{V}_{x}-V_{0}\right)\left(\bar{V}_{p}-V_{0}\right) \geq\left(2 V_{0}\right)^{2}$ states that the product of the extra noises is not less than $\left(2 V_{0}\right)^{2}$. This $2 V_{0}$ coincides with two quduties [10] which have been introduced as the extra noise induced by the classical teleportation [21, 30]. However, note that our bound $\left(\bar{V}_{x}-V_{0}\right)\left(\bar{V}_{p}-V_{0}\right) \geq\left(2 V_{0}\right)^{2}$ reveals a more fundamental aspect of two quduties, that is, two quduties $2 V_{0}$ correspond to the minimum of extra noises induced by arbitrary EB channels. Moreover, the role of the product form is striking. In general we could observe $\bar{V}_{x} \neq \bar{V}_{p}$, and some of EB maps induce an extra noise for one quadrature, say $\hat{x}$, so that it keeps below two quduties as $\bar{V}_{x}-V_{0}<2 V_{0}$. However, even in such cases, our formula states that the extra noise of the other quadrature $\bar{V}_{p}-V_{0}$ has to increase to fulfill the limit in the product form. Therefore, the classical penalty on the canonical variables is demonstrated as a fundamental basis through the uncertainty product and it refines the notion of two quduties. This fundamental structure holds for non-unit gain $\eta>0$ and partially known coherent states $\lambda>0$. A non-unity gain $\eta \neq 1 \mathrm{im}$ plies the amplitude transformation $\alpha \rightarrow \sqrt{\eta} \alpha$. Thereby, the minimum of the uncertainty product has to keep the scaling determined by the gain factor $\eta$ similarly to the amplification-uncertainty principle [40]. For a finite distribution, $\lambda^{-1}$ represents the width of the prior $p_{\lambda}$ of Eq. (8). Hence, the factor $1+\lambda$ of Eq. (6) is thought to be the reduction of the uncertainty due to the amount of prior knowledge.

Interestingly, one can find an EB map that achieves the equality of Eq. (6) for any possible parameter set of $(\eta, \lambda)$. This means that Eq. (6) is tight for every pair of $(\eta, \lambda)$ and the inversely proportional curves of Fig. 1 entirely describe an optimal trade-off relation between canonical quantum noises to beat the classical channels. In fact, we can show Eq. (6) is saturated by the EB map of

$$
\mathcal{E}_{M P}(\rho)=\int S_{R}|\gamma \alpha\rangle\left\langle\alpha\left|S_{r}^{\dagger} \rho S_{r}\right| \alpha\right\rangle\langle\gamma \alpha| S_{R}^{\dagger} \frac{d^{2} \alpha}{\pi},
$$

where $S_{r}=e^{r\left(\hat{a}^{2}-\hat{a}^{\dagger 2}\right) / 2}$ is a squeezer and

$$
\begin{aligned}
\gamma & =\frac{\sqrt{\eta}}{\sqrt{(1+\lambda)^{2} \cosh ^{2} r-\sinh ^{2} r}}, \\
e^{R} & =\sqrt{\frac{(1+\lambda) \cosh r+\sinh r}{(1+\lambda) \cosh r-\sinh r}} .
\end{aligned}
$$

This yields a simple form $\left(\bar{V}_{x}, \bar{V}_{p}\right)=u^{-2}\left(e^{-2 R}+v^{2}, e^{2 R}+\right.$ $\left.v^{2}\right) / 2$ with Eq. (13), and $R$ determines the balance between $\bar{V}_{x}$ and $\bar{V}_{p}$. Obviously, $\mathcal{E}_{M P}$ represents the channel that prepares a minimum uncertainty state after a projection to a minimum uncertainty state. This structure demonstrates the mechanism that each of the measure and preparation processes is responsible for increasing the excess noises by a single quduty.

As we will prove next, our QB is enough to detect all one-mode Gaussian channels in the quantum domain. This is reasonable because $J$ is a Gaussian state whenever $\mathcal{E}$ is a Gaussian channel and the separable condition of Eq. (1) is known to be sufficient for detection of any two-mode Gaussian entanglement. In the proof of Proposition, our input ensemble $\left\{p_{\lambda}(\alpha), \rho_{\alpha}\right\}_{\alpha \in \mathbb{C}}$ is determined by the pair of the two-mode squeezed state $\left|\psi_{\xi}\right\rangle$ and the

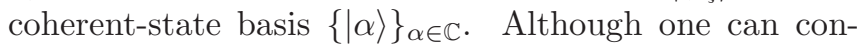
sider different input ensembles by assigning other entangled states, it remains open whether any given ensemble can be related to a meaningful entanglement detection scenario [41]. On the other hand, the basis $\{|\alpha\rangle\}_{\alpha \in \mathbb{C}}$ is rather regarded as a choice of the representation that executes the partial trace, but, enables us to introduce experimentally relevant input states.

We can show the converse statement of our Proposition for the class of one-mode Gaussian channels: If $\mathcal{E}$ is Gaussian and not EB, there exists a set of $(\eta, \lambda)$ and additional Gaussian unitary operators so that $\mathcal{E}$ violates Eq. (6). This can be proven similarly to the case of the fidelity-based benchmark [5]: Thanks to Holevo's 
classification of Gaussian channels [42], it is sufficient to check that the following two types of the channels violate Eq. (6). One is a unit-gain channel $(\eta=1)$ which adds one unit of shot noise to one of quadratures, e.g., $\left(\bar{V}_{x}, \bar{V}_{p}\right)=V_{0}(2,1)$. It violates the condition of Eq. (6) for $\lambda<4$. The other is an amplification/attenuation channel which transforms the moments of both quadratures as $z_{\alpha} \rightarrow \sqrt{G} z_{\alpha}$ and $\left\langle\hat{z}^{2}\right\rangle_{\rho_{\alpha}} \rightarrow G z_{\alpha}^{2}+\tilde{n}+(G+|1-G|) / 2$ where $G \geq 0$ is an actual gain and $\tilde{n} \in[0, \min \{1, G\})$. This implies $\bar{V}_{z}=\lambda^{-1}(\sqrt{G}-\sqrt{\eta})^{2}+\tilde{n}+(G+|1-G|) / 2$, and the condition of Eq. (6) is violated if $(\eta, \lambda)=(4 G, 1)$.

Note that, if the channel is assumed to be Gaussian, it is covariant under displacement [6, 43]. Then, one can determine any channel parameters through covariance matrices based on input of a single coherent state. However, the displacement covariance is not physically justifiable because it implies that the channel maintains a linear response even for any high energy input state. Hence, we are better off using the Gaussian assumption for channels. In our theorem, the footing of Gaussian distributed coherent states bypasses the Gaussian assumption and gives us a practical platform to explore effectively linear responses [34, 38]. Such a framework would be crucial in experiments to deal with Gaussian and non-Gaussian ingredients equally well.

While the product form of uncertainties represents a fundamental boundary, an EB bound focusing on the total quadrature noise $\bar{V}:=\bar{V}_{x}+\bar{V}_{p}$ was known in Ref. [5]. We can improve this bound as a corollary.

Corollary 1.- - Let us define the total noise as $\bar{V}:=$ $\bar{V}_{x}+\bar{V}_{p}$ with Eq. (7). For any $\lambda>0$ and $\eta>0$, any EB channel $\mathcal{E}$ satisfies

$$
\frac{\bar{V}}{2}=\frac{\bar{V}_{x}+\bar{V}_{p}}{2} \geq \frac{1}{2}+\frac{\eta}{1+\lambda} .
$$

This can be proven by applying the relation $|a|+|b| \geq$ $2 \sqrt{|a b|}$ to Proposition. The inequality of Eq. (17) is tight as it can also be saturated by the EB map $\mathcal{E}_{M P}$ of Eq. (15) with $r=0$. It improves the QB inequality of Eq. (10) in Ref. [5] (See [44]). Corollary 1 can be associated with the famous sum condition for separability [32] whereas Proposition has its origin in the product separable condition of Eq. (5). Note that, from the total noise $\bar{V}$, one can obtain a lower bound of the average fidelity $\bar{F}_{\eta \cdot \lambda}:=$ $\int p_{\lambda}(\alpha)\left\langle\sqrt{\eta} \alpha\left|\mathcal{E}\left(\rho_{\alpha}\right)\right| \sqrt{\eta} \alpha\right\rangle d^{2} \alpha$ [4, 5, 21, 34, 38, 39] by using the relation $\bar{F}_{\eta, \lambda} \geq(3-\bar{V}) / 2$ introduced in [5]. This supports the intuition that a smaller excess noise implies a higher fidelity, and simply connects the measurement of $\left(\bar{V}_{x}, \bar{V}_{p}\right)$ to an estimation of the fidelity.

Finally, we generalize our Proposition to address the effects of (i) asymmetric gains where the first moments are expected to transform $\left(x_{\alpha}, p_{\alpha}\right) \rightarrow\left(g_{x} x_{\alpha}, g_{p} p_{\alpha}\right)$ [19, 20] and (ii) the post-selection where the channel can be a trace-decreasing map (stochastic quantum channel) [34].

Corollary 2.-For any $\lambda>0$ and any gain pair $\left(g_{x}, g_{p}\right)>0$, any stochastic EB map $\mathcal{E}$ satisfies

$$
\left[\tilde{V}_{x}-\frac{g_{x}^{2}}{2(1+\lambda)}\right]\left[\tilde{V}_{p}-\frac{g_{p}^{2}}{2(1+\lambda)}\right] \geq \frac{1}{4}\left(1+\frac{g_{x} g_{p}}{1+\lambda}\right)^{2}
$$

where $\tilde{V}_{z}:=\bar{V}_{z}\left(g_{z}^{2}, \lambda\right) / \operatorname{Tr}\left[\int p_{\lambda}(\alpha) \mathcal{E}\left(\rho_{\alpha}\right) d^{2} \alpha\right]$ with Eq. (7).
To prove Corollary 2, we replace $J$ in Eq. (11) with $\left(S_{q}\right)_{B} J\left(S_{q}^{\dagger}\right)_{B}$. This transforms the quadratures in Lemma as $\left(\hat{x}_{A}, \hat{p}_{A}\right) \rightarrow\left(\hat{x}_{A} e^{q}, \hat{p}_{A} e^{-q}\right)$. Further, repeating the proof of Proposition starting with $J=\mathcal{E}_{A} \otimes$ $I_{B}\left(\left|\psi_{\xi}\right\rangle\left\langle\psi_{\xi}\right|\right) / \operatorname{Tr}\left[\mathcal{E}_{A} \otimes I_{B}\left(\left|\psi_{\xi}\right\rangle\left\langle\psi_{\xi}\right|\right)\right]$ instead of Eq. (9) we can reach Corollary 2 with the form of the gain pair $\left(g_{x}, g_{p}\right)=\left(\sqrt{\eta} e^{-q}, \sqrt{\eta} e^{q}\right)$. Since the underlying physics does not change as long as $J$ is normalized, $\mathcal{E}$ is not necessary to be a trace-preserving operation. Thus, EB channels are unable to beat our bound even stochastically. Therefore, Corollary 2 constitutes a unified QB that works with feasible input-and-measurement settings for a wide class of CV channels by assigning a gain pair $\left(g_{x}, g_{p}\right)$. Corollary 2 also describes an optimal trade-off relation due to EB maps since the inequality of Eq. (18) is saturated by the EB channel $\mathcal{E}_{M P}^{\prime}(\rho)=S_{q} \mathcal{E}_{M P}(\rho) S_{q}^{\dagger}$ with Eq. (16) for any given $\left(g_{x}, g_{p}, \lambda\right)>0$. Although one can use the fidelity-based QB [5] for asymmetric gains, it may require a type of squeezed resources such as a measurement of the fidelities to squeezed states [18].

In conclusion, we have established an uncertaintyrelation-type QB for CV quantum channels. It is usable to verify the quantum-domain performance for a wide class of $\mathrm{CV}$ quantum channels by assigning a pair of quadrature gains including stochastic quantum channels. Our results generally explain the classical penalty of two quduties and an optimal trade-off relation on canonical variables to beat EB channels. This highlights a structural difference from the fidelity-based QB [5]. We have also proven the converse statement of our QB for onemode Gaussian channels. Hence, our framework has no less generality than the framework of the fidelity-based QB. It would be fundamental to address the quantumamplification limit [34, 38, 40] and related cloning limits in our canonical basis [45]. Although we have concentrated on a single separable condition of Eq. (1), one can use our approach to translate a wide class of separable conditions [46 48] into quantum benchmarking conditions [49].

We thank N. Lütkenhaus for helpful discussions. This work was partly supported by GCOE Program "The Next Generation of Physics, Spun from Universality and Emergence" from MEXT of Japan and World-Leading Innovative R\&D on Science and Technology (FIRST). $\mathrm{KA}$ is in part supported by the Project UQCC by the National Institute of Information and Communications Technology (NICT).

* Electric address: namiki@scphys.kyoto-u.ac.jp

[1] S. Popescu, Phys. Rev. Lett. 72, 797 (1994).

[2] S. Massar and S. Popescu, Phys. Rev. Lett. 74, 1259 (1995).

[3] M. Horodecki, P. Horodecki, and R. Horodecki, Phys. Rev. A 60, 1888 (1999).

[4] K. Hammerer, M. M. Wolf, E. S. Polzik, and J. I. Cirac, Phys. Rev. Lett. 94, 150503 (2005).

[5] R. Namiki, M. Koashi, and N. Imoto, Phys. Rev. Lett. 101, 100502 (2008).

[6] M. Owari et al., New J. Phys. 10, 113014 (2008). 
[7] J. Calsamiglia, M. Aspachs, R. Munoz-Tapia, and E. Bagan, Phys. Rev. A 79, 050301 (2009).

[8] H. Häseler and N. Lütkenhaus, Phys. Rev. A 81, 060306(R) (2010).

[9] M. Horodecki, P. W. Shor, and M. B. Ruskai, Rev. Math. Phys. 15, 629 (2003).

[10] A. Furusawa et al., Science 282, 706 (1998); S. L. Braunstein and H. J. Kimble, Phys. Rev. Lett. 80, 869 (1998).

[11] B. Julsgaard, J. Sherson, J. I. Cirac, J. Fiurásek, and E. S. Polzik, Nature (London) 432, 482 (2004); K. Jensen et al., Nat. Phys. 7, 13 (2011).

[12] R. Namiki and Y. Tokunaga, Phys. Rev. A 85, 010305(R) (2012).

[13] R. Namiki and T. Hirano, Phys. Rev. Lett. 92, 117901 (2004).

[14] J. Rigas, O. Gühne, and N. Lütkenhaus, Phys. Rev. A 73, 012341 (2006).

[15] N. Killoran, H. Häseler, and N. Lütkenhaus, Phys. Rev. A 82, 052331 (2010); N. Killoran and N. Lütkenhaus, Phys. Rev. A 83, 052320 (2011); N. Killoran, M. Hosseini, B. C. Buchler, P. K. Lam, and N. Lütkenhaus, Phys. Rev. A 86, 022331 (2012).

[16] I. Khan, C. Wittmann, N. Jain, N. Killoran, N. Lütkenhaus, C. Marquardt, and G. Leuchs, Phys. Rev. A 88, 010302 (2013).

[17] C. A. Fuchs and M. Sasaki, Quantum Inf. Comput. 3, 377 (2003).

[18] R. Namiki, Phys. Rev. A 78, 032333 (2008).

[19] W. P. Bowen, N. Treps, B. C. Buchler, R. Schnabel, T. C. Ralph, T. Symul, and P. K. Lam, IEEE J. Sel. Top. Quantum Electron. 9, 1519, (2003).

[20] T. C. Ralph and P. K. Lam, Phys. Rev. Lett. 81, 5668 (1998); W. Bowen et al., Phys. Rev. A 67, 032302 (2003).

[21] S. L. Braunstein, C.A. Fuchs, and J. Kimble, J. Mod. Opt. 47, 267 (2000).

[22] F. Grosshans et al., Quant. inf. Comp., 3535 (2003).

[23] G. Hétet, A. Peng, M. T. Johnsson, J. J. Hope, and P. K. Lam, Phys. Rev. A 77, 012323 (2008).

[24] S. L. Braunstein and P. van Loock, Rev. Mod. Phys. 77, 513 (2005); N. J. Cerf, G. Leuchs, and E. S. Polzik (eds), Quantum Information with Continuous Variables of Atoms and Light (Imperial College Press, 2007); C. Weedbrook et al., Rev. Mod. Phys. 84, 621 (2012).

[25] A. I. Lvovsky, B. C. Sansers, and W. Tittel, Nat. Photon. 3, 706 (2009); K. Hammerer, A. S. Sorensen, and E. S. Polzik, Rev. Mod. Phys. 82, 1041 (2010); U. L. Andersen, G. Leuchs, and C. Silberhorn, Laser \& Photonics Reviews 4, 337 (2010).

[26] T. Takano, M. Fuyama, R. Namiki, and Y. Takahashi, Phys. Rev. Lett. 102, 033601 (2009); T. Takano, S. Tanaka, R. Namiki, and Y. Takahashi, Phys. Rev. Lett. 104, 013602 (2010); R. Inoue, S. Tanaka, R. Namiki, T. Sagawa, and Y. Takahashi, Phys. Rev. Lett. 110, 163602 (2013).
[27] E. Arthurs and J. L. Kelly, Jr., Bell System Tech. J. 44,725 (1965); S. Stenholm, Ann. Phys. 218, 233 (1992).

[28] See, e.g., Sec. 6 of U. Leonhardt, Measuring the Quantum State of Light (Cambridge, 1997).

[29] Note that, in the case of simultaneous measurements, the noise penalty is given through a generalized canonical uncertainty relation and a single quduty represents an accurate trade-off relation.

[30] Note that the noise penalty of two quduties is given by an EB channel implemented by heterodyne measurement and preparation of coherent states [21]. This model of measure-and-prepare schemes, commonly termed as the classical teleportation, constitutes an optimal EB map for the fidelity-based QBs [4, 5] and gives an optimal amount of noise tolerance for an entanglement transmission through a generic Gaussian channel (See Eq. (4) of Ref. [8]).

[31] Roughly speaking, Eq. (10) of Ref. [5] shows that a single quduty works as a QB.

[32] L.-M. Duan, G. Giedke, J. I. Cirac, and P. Zoller, Phys. Rev. Lett. 84, 2722 (2000).

[33] R. Simon, Phys. Rev. Lett. 84, 2726 (2000).

[34] G. Chiribella and J. Xie, Phys. Rev. Lett. 110, 213602 (2013).

[35] V. Giovannetti, S. Mancini, D. Vitali, and P. Tombesi, Phys. Rev. A 67, 022320 (2003).

[36] R. Namiki, J. Phys. Soc. Jpn. 82, 014001 (2013).

[37] G. M. D'Ariano, P. Kumar, C. Macchiavello, L. Maccone, and N. Sterpi, Phys. Rev. Lett. 83, 2490 (1999).

[38] R. Namiki, Phys. Rev. A 83, 040302(R) (2011).

[39] R. Namiki, Phys. Rev. A 83, 042323 (2011).

[40] C. M. Caves, Phys. Rev. D 26, 1817 (1982).

[41] Note that there is a group-theoretical approach to provide a general prior to introduce a fidelity-based $\mathrm{QB}$ for input of pure single-mode Gaussian states. See G. Chiribella and G. Adesso, Phys. Rev. Lett. 112, 010501 (2014).

[42] A. S. Holevo, Probl. Inf. Transm. 44, 171 (2008).

[43] M. Guta, P. Bowles, and G. Adesso, Phys. Rev. A 82, 042310 (2010).

[44] A similar sum condition appears in [20] and coincides with Eq. (17) in the limit $\lambda \rightarrow 0$. However, the results in Ref. 20] are based on the conditional variance with some other constraints, and it is an open question whether one can find a rigorous link to our result.

[45] R. Namiki, arXiv:1502.05031

[46] E. Shchukin and W. Vogel, Phys. Rev. Lett. 95, 230502 (2005).

[47] A. Miranowicz, M. Piani, P. Horodecki, and R. Horodecki, Phys. Rev. A 80, 052303 (2009).

[48] R. Namiki, Phys. Rev. A 85, 062307 (2012).

[49] R. Namiki, arXiv:1503.07109 